\title{
Index rerum ad Vol. 170
}

\section{Confecit: C. Loeb-Schürch}

$(\mathrm{V})=$ Report - Vortrag - Communication $(\mathrm{B})=$ Book Reviews-Buchbesprechungen-Livres nouveaux

Abfiussfazilität, v. Propanololum Ablatio, v. Adaptométrie, Retinoschisis Abszess, v.

Endocarditis Acuité visuelle, v. Gesichtsfeldausfälle Acuité visuelle en profondeur, v. Depth acuity Adaptométrie et périmétrie statique dans

les temps postopératoires immédiats du

décollement de la rétine 450 Adenosindiphosphat, v. Microcirculation Aderhaut, v.

Choroïdopathie maculaire,

Sarkoidose, Tuberculose miliaire ADP, v. Microcirculation Aides électroniques, v.

Farbidentifizie-

rungsgerät, Hilfsmittel Akkommodation, v. Mehrstärkenglas,

Zentralskotom Alcali-burn, v. Homogenisates Amblyopie, v. Kongenitales Glaukom,

Polatest American Ophthalmological Society;

transactions of the American Ophthalmological Society 475 (B) Amyloid, v. Temporal arteritis

Amyloid und Auge 180 (V) Anesthesia; corneal anesthesia induced

by soaps and surfactants. Lack of

correlation in rabbits and humans 320 Angiitis, v. Sarkoidose, Temporal

arteritis Angioscopy, v. Color angiography Anomalies constitutionnelles v. Retino-

pathia pigmentosa

Anomalies d'insertion, v. Ophtalmoplégie Anterior chamber, v. Tolerance, Vorder-

kammerlinsen Antibiotica, v. Endocarditis, Yeasts Antibiotique; etude et résultats d'un

traitement antibiotique standardise

après plaie perforante du globe

oculaire 174 (V) Antigène australien, v. Immunologie Antimitotiques, v. Tolerance Aplasie musculaire, v. Ophtalmoplégie Apocrine mixed tumour, v. Mixed tumour Aqueous humor, v. Propanololum Arterial pressure, v. Migraine Arteriitis temporalis, v. Temporal arteritis Atrophie optique, v. Retinopathia

pigmentosa, Vascularisation papillaire Augenbinnendruck, v. Propanololum, Tonomètre Augenmuskeln, v. Ophtalmoplégie,

Strabismus sursoadductorius Australia-Antigen, v. Immunologie Autoimmunkrankheiten, v. Immunologie Automation, v. Examen automatique Automatisierung der Perimetrie 106 (V) Autoradiographie, v. Wound healing

Basaliom, v. Langerhans-Granula Bâtonnets, v. Netzhautfunktionen Bestrahlung, v.

Papillenschwellungen,

Temperaturen, Thromben Bindehaut, v. Amyloid, Neurodermitis

disseminata, Yeasts

Index re rum ad Vol. 170

555 
Binokularsehen, v. Depth acuity,

Equilibre, Polatest Biochemie, v. Transferrine Blepharitis, v. Yeasts Blindness, v.

Farbidentifizierungsgerät,

Hilfsmíttel Blutdruck, v. Migraine Boeck’sches Sarkoid, v. Sarkoidose Book reviews 84(B), 392(B),

475 (B) Bovine, v. Homogenisates Brille, v. Mehrstärkenglas, Polatest Bruch'sche Membran, v.

Choroïdopathie

maculaire Brûlure, v. Homogenisates Buchbesprechungen 84 (B), 392 (B),

475 (B) Buphthalmus, v. Juveniles Glaukom,

Kongenitales Glaukom, Trisomie-13-

Syndrom

Calcifications pulmonaires, v. Choroïdo-

pathie maculaire Cancroïde, v. Langerhans-Granula Candidase, v. Yeasts Capillary permeability,

v. Dobésilate de

calcium, Exsudative Retinopathie Capillary resistance, v. Circulatory

disorders, Dobésilate de calcium,

Medikamentöse Therapie Cataracte, v. Iridectomie filtrante, Micro-

chirurgie, Tolerance, Vorderkammer-

linsen Catécholamines, v. Horner's syndrome Cécité, v. Farbidentifizierungsgerät, Hilfs-

mittel Chambre antérieure, v. Tolerance,

Vorderkammerlinsen Champ visuel, v. Automatisierung,

Gesichtsfeldausfälle, Netzhaut-

funktionen Chat, v. Tolerance Chien, v. Netzhautatrophie Chirurgie, v. Adaptométrie,

iridectomie

filtrante, Irisnaht, Juveniles Glaukom,

Kongenitales Glaukom, Micro-chirurgie, Pathologische Befunde,

Pupillenerhaltung, Strabismus surso-adductorius, Trabéculectomie, Trabé-culotomie,

Trabekulektomie, Tumeurs multiples Chorioidea, v. Choroïdopathie maculaire.

Sarkoidose, Tuberculose miliaire Chorioretinal degeneration, v. Netzhaut

funktionen Choroïdopathie maculaire hémorragique

chez les sujets jeunes 477 Chromosom, v. Retinoblastoma,

Trisomie-13-Syndrom Chymotrypsin, v. Micro-chirurgie Cicatrisation, v. Wound healing Ciliary body, v. Metastatic tumour,

Sarkoidose Circulatory disorders; effect of calcium dobésilate (Doxium ${ }^{\circledR}$ ) on circulatory

disorders of the retina with special emphasis on diabetic retinopathy 434 Clear cell hidradenoma,

v. Mixed tumour Clinicopathologic case reports 381, 543,

548 Clostridium perfringens, v. Gasbrand Coagulation, v. Fibrinolytic system,

Microcirculation Cocain, v. Horner's syndrome Color angiography and angioscopy 426 Color

vision, v. Dyschromatopsy Complications oculaires, v. Iridectomie filtrante, Micro-chirurgie,

Neuro-dermitis disseminata, Trabéculectomie, Trabéculotomie, Vorderkammerlinsen Cones, v.

Netzhautfunktionen Congrès, v. Société Suisse d'Ophtalmo-

logie Conjunctiva, v. Amyloid, Neurodermitis

disseminata, Yeasts Conjunctivitis, v. Yeasts Contact lenses, v. Soft lens Convergence, v.

Mehrstärkenglas Cornea, v. Anesthesia, Fusarium, Homogenisates, Hornhautdystrophie,

Juveniles Glaukom, Micro-chirurgie, Neurodermitis disseminata, Soft lens, Yeasts 
556

Index rerum ad Vol. 170

Corneal sensitivity, v. Anesthesia Corps ciliaire, v. Metastatic tumour,

Sarkoidose Corps étranger, v. Antibiotique Corps vitré, v. Fibroplasie, Pars planitis,

Vitrektomie Cottonwool spots, v. Exsudative Retino-

pathie Cristallin, v. Micro-chirurgie, Trisomie-

13-Syndrom, Vorderkammerlinsen Cryochirurgie, v. Micro-chirurgie Cystoid degeneration, v.

Retinoschisis

Dacryocanaliculitis, v. Yeasts

Deafness, v. Retinopathia pigmentosa

Décollement rétinien, v. Adaptométrie, Retinoschisis

Dégénérescence kystoïde, v. Retinoschisis

Dégénérescence rétinienne, v. Netzhaut-atrophie, Netzhautfunktionen, Retinopathia pigmentosa

Denervation supersensitivity, v. Horner's syndrome

Depth acuity; relationship between monocular and binocular depth acuity 43

Desoxyribonukleinsäure, v. Wound healing

Detergents, v. Anesthesia

Diabetic retinopathy, v. Circulatory disorders, Dobésilate de calcium, Exsudative Retinopathie, Fibrinolytic system, Medikamentöse Therapie, Microcirculation, Verfettung

Diagnostic, v. 3äP-Test, Retinoschisis, Tumeurs intra-oculaires

Diagnostic étiologique des uvéites: contribution du test au nitrobleu de tétrazolium 192 (V)

Diamètre cornéen, v. Kongenitales Glaukom

DNA, v. Wound healing

Dobésilate de calcium; cinq années d'ex-périmentation avec le dobésilate de calcium dans le traítement des rétino-pathies $232(\mathrm{~V})$

Dog, v. Netzhautatrophie

Doxium, v. Circulatory disorders,

Dobésilate de calcium, Medikamentöse

Therapie Dunkeladaptation, v. Adaptométrie Dyschromatopsy and unilateral glaucoma

30 Dystrophie cornéenne, v. Hornhaut-

dystrophie

Echographie, v. Tumeurs intra-oculaires EEG, v. Retinopathia pigmentosa Ektopia retinae, v.

Trisomie-13-Syndrom Electronic aids, v. Farbidentifizierungs-

gerät, Hilfsmittel Elektroenzephalogramm, v. Retinopathia

pigmentosa Elektromyogramm, v. Retinopathia

pigmentosa Elektronenmikroskopie, v. Homogeni-

sates, Hornhautdystrophie, lrisnaht,

Langerhans-Granula, Wound

healing Elektroretinogramm, v. Netzhautfunktionen, Retinopathia pigmentosa Elliott'sche

Trepanation, v. Juveniles

Glaukom EMG, v. Retinopathia pigmentosa Endocarditis; prepapillary metastatic

abscess in a case of subacute bacterial

endocarditis 22 Enzephalomeningitis chronica dissemi-

nata, v. Sarkoidose Epitheliom, v. Langerhans-Granula Epithelium pigmentaire, v. Wound

healing Equilibre; importance des examens 
oculo-moteurs dans les troubles de

l'équilibre 280 (V) ERG, v. Netzhautfunktionen, Retinopathia pigmentosa Examen automatique; reactions psycho-

logiques du patient soumis à un

examen ophtalmologique automatique

88 (V) Examen oculo-moteur, v. Equilibre Experimentelle Forschung, v. Anesthesia,

Depth acuity, Homogenisates, lrisnaht,

Index rerum ad Vol. 170

557

Microcirculation, Tolerance, Trabe-

kulopunktur, Transferrine, Wound

healing, Yeasts Exsudative Retinopathie; die exsudative

diabetische Retinopathie 518 Exsudats durs, v. Exsudative Retinopathie

Facilité d'écoulement, v. Propanololum

Farbidentifizierungsgerät für Blinde 130 (V)

Farbsinn, v. Dyschromatopsy

Fibrinolytic system; the fibrinolytic system in patients with diabetes mellitus with special reference to diabetic retinopathy 353

Fibroplasie; spontane epiretinale Fibro-plasie 217 (V)

Filter, v. Color angiography, Vascularisation papillaire

Fluoro-Angiographie, v. Circulatory disorders, Choroïdopathie maculaire, Color angiography, Exsudative Retinopathie, Fibroplasie, Microcirculation, Netzhautfunktionen, Pars planitis,

Tuberculose miliaire, Vascularisation papillaire

Foreign body, v. Antibiotique

Fremdkörper, v. Antibiotique

Fundus, v. Ophthalmoscope

Fusarium; mycotic keratitis by fusarium. A review and report of two cases 1

Gangrene gazeuse, v. Gasbrand

Gasbrand; Befunde am Augenhinter-grund bei Gasbrand 334

Gefässe, v. Choroïdopathie maculaire, Circulatory disorders, Color angiography, Dobésilate de calcium, Exsudative Retinopathie, Fibrinolytic system, Gasbrand, Medikamentöse Therapie, Microcirculation, Pars planitis, Temperaturen, Temporal arteritis, Thromben, Vascularisation papillaire, Verfettung

Genetics, v. Amyloid, Netzhautatrophie, Netzhautfunktionen, Neurodermitis disseminata, Ophtalmoplégie, Retino-

blastoma, Retinopathia pigmentosa,

Temporal arteritis Gerinnung, v. Fibrinolytic system,

Microcirculation Gesichtsfeld, v. Automatisierung,

Netzhautfunktionen Gesichtsfeldausfälle; Problematik der

Sehfunktion bei parazentralen Ge-

sichtsfeldausfällen 261 (V) Glande lacrymale, v. Tumeurs

lymphoïdes Glande sudoripare, v. Mixed tumour Glaskörper, v. Fibroplasie, Pars planitis, Vitrektomie Glasses, v. Mehrstärkenglas, Polatest Glaucoma, v. Dyschromatopsy, Iridectomie filtrante, Juveniles Glaukom,

Kongenitales Glaukom, Pathologische 
Befunde, Propanololum, Trabéculec-

tomie, Trabéculotomie, Trabekulek-

tomie, Trabekulopunktur Glaukom 475 (B) Glaukom in der Praxis 392 (B) Gleichgewicht, v. Equilibre Gleitsichtglas, v. Mehrstärkenglas Gliclazid, v. Microcirculation Globuline, v. Immunologie Goniolyse, v. Kongenitales Glaukom Goniotomie, v. Juveniles Glaukom, Kongenitales Glaukom Granulom, v. Sarkoidose

Headache, v. Equilibre, Migraine,

Polatest Hefen, v. Yeasts

Hemorrhagie rétinienne, v. Endocarditis Hepatitis, v. Immunologie Hérédité, v. Amyloid, Netzhautatrophie,

Netzhautfunktionen, Neurodermitis

disseminata, Ophtalmoplégie, Retino-

blastoma, Retinopathia pigmentosa,

Temporal arteritis Heterophorie, v. Equilibre Hidradenoma, v. Mixed tumour Hilfsmittel;

elektronische Hilfsmittel für

Blinde und hochgradig Sehschwache

$94(\mathrm{~V})$

558

Index rerum ad Vol. 170

Histologie, v. Fusarium, Metastatic tumour, Mixed tumour, Netzhaut-atrophie, Neurodermitis disseminata, Pathologische Befunde, Sarkoidose, Temporal arteritis, Trisomie-13-Syndrom, Tumeurs intra-oculaires, Tumeurs lymphoïdes, Tumeurs multiples, Wound healing

Histoplasmose, v. Choroïdopathie maculaire

Homogenisates; scanning microscope observations of alcali-burned rabbit cornea treated with embryonic corneal homogenisates 64

Horner's syndrome; denervation super-sensitivity in Horner's syndrome 313

Hornhaut, v. Anesthesia, Fusarium, Homogenisates, Hornhautdystrophie, Juveniles Glaukom, Micro-chirurgie, Neurodermitis disseminata, Soft lens, Yeasts

Hornhautdurchmesser, v. Kongenitales Glaukom

Hornhautdystrophie; Ultrastruktur der gittrigen Hornhautdystrophie 191 (V)

SH-Thymidin, v. Wound healing

Humeur aqueuse, v. Propanololum

Hund, v. Netzhautatrophie

Hyaloretinitis, v. Pars planitis

Hypernephroma, v. Metastatic tumour

Hyperuricaemie, v. Retinopathia pigmentosa

Hypotension orthostatique, v. Migraine

Immunologíe; apport de Гimmunologie de routine au bilan de Гuvéite 193 (V)

Infektion, v. Endocarditis, Gasbrand, Tuberculose miliaire

Inflammation, v. Endocarditis, Fusarium, Pars planitis, Temporal arteritis, Yeasts

Inflammations; ocular inflammat. 84 (B)

Innenohrschwerhörigkeit, v. Retinopathia pigmentosa

Insertions-Anomalien, v. Ophtalmoplegie

Instrument, v. Color angiography, Juveniles Glaukom, Ophthalmoscope, 32P-test, Tonomètre, Vitrektomie 
Iridectomie filtrante; six ans d'expérience

de Tiridectomie filtrante 146 (V) Iris, v. Iridectomie filtrante, Pupillen-

erhaltung. Tumeurs multiples Iris-clip-lens, v. Vorderkammerlinsen Irisnaht. Experimentelle und klinische

Resultate 150 (V) Irisprolaps, v. Pupillenerhaltung Iritis, v. Sarkoidose Irradiation, v.

Papillenschwellungen

Temperaturen, Thromben

Juveniles Glaukom; operative Behand-lung des kindlichen und juvenilen Glaukoms 139 (V)

Kammerwasser, v. Propanololum Kaninchen, v. Anesthesia, Homogenisates,

Irisnaht, Transferrine, Wound healing Kapillarpermeabilität, v. Dobésilate de

calcium, Exsudative Retinopathie Kapillarresistenz, v. Circulatory disorders,

Dobésilate de calcium, Medikamentöse

Therapie Katarakt, v. Iridectomiefiltrante, Microchi-

rurgie, Tolerance, Vorderkammerlinsen Katecholamine, v. Horner's syndrome Katze, v.

Tolerance Keratitis, v. Fusarium, Yeasts Keratomycosis, v. Fusarium Komplikationen, v.

Iridectomie filtrante,

Micro-chirurgie, Neurodermitis disseminata, Trabéculectomie, Trabéculo-

tomie, Vorderkammerlinsen Kongenitales Glaukom; Behandlungs-

resultate bei 45 Kindern mit kongeni-

talem Glaukom 115 (V) Konstitutionsanomalien, v. Retinopathia

pigmentosa Kontaktlinsen, v. Soft lens Konvergenz, v. Mehrstärkenglas Kopfschmerz, v.

Equilibre, Migraine,

Polatest Kryochirurgie, v. Micro-chirurgie Lacrimal gland, v. Tumeurs lymphoïdes Lacrimal pathways, v. Yeasts Lähmung, v. Equilibre, Ophtalmoplegie

Index rerum ad Vol. 170

559

Langerhans-Granula in Epitheliomen der Lider 534

Langzeittherapie; wann soil der Augen-arzt in der Praxis eine Langzeittherapie abbrechen? 309

(V)

Lapin, v. Anesthesia, Homogenisates, Irisnaht, Transferrine, Wound healing

Laser, v. Trabekulopunktur

Lehrbuch und Atlas der Augenheilkunde 84(B)

Lens, v. Micro-chirurgie, Trisomie-13-Syndrom, Vorderkammerlinsen

Lentilles de contact, v. Soft lens

Lesegerät, v. Hilfsmittel

Leukaemie, v. Papillenschwellungen

Levures, v. Yeasts

Lid, v. Langerhans-Granula, Mixed tumour, Tumeurs multiples, Yeasts

Linse, v. Micro-chirurgie, Trisomie-13-Syndrom, Vorderkammerlinsen

Lipoidherde, v. Exsudative Retinopathie

Lipoproteine, v. Retinopathia pigmentosa

Liquor cerebrospinalis, v. Papillenschwellungen, Retinopathia pigmentosa

Livres nouveaux 84 (B), 392 (B), 475 (B)

Lowe-Syndrom, v. Trisomie-13-Syndrom

Lufttonometer, v. Tonomètre 
Lunettes, v. Mehrstärkenglas, Polatest

Lungenverkalkungen, v. Choroïdopathie maculaire

Macula, v. Adaptométrie, Choroïdopathie maculaire, Endocarditis

Makulaoedem, v. Pars planitis

Malformations, v. Juveniles Glaukom, Kongenitales Glaukom, Ophtalmo-plégie, Trisomie-13-

Syndrom

Maux de tête, v. Equilibre, Migraine, Polatest

Medikamentöse Therapie; Sinn und Unsinn medikamentöser Therapie bei Retinopathia diabetica $239(\mathrm{~V})$

Mehrstärkenglas; ein neues Mehrstärkenglas 370

Melanoma, v. Tumeurs intra-oculaires

Meningen, v. Papillenschwellungen, Sarkoidose

Meningitis, v. Sarkoidose

Métabolisme, v. Retinopathia pigmentosa

Metastatic tumour of the ciliary body -hypernephroma 543

Methode, v. Automatisierung, Color angiography, Depth acuity, Examen automatique,

Farbidentifizierungsgerät, Hilfsmittel, lridectomie filtrante, Irisnaht, Juveniles Glaukom, Micro-

chirurgie, Polatest, 32P-Test, Pupillen-erhaltung, Vascularisation papillaire,

Vorderkammerlinsen

Microangiopathie, v. Circulatory disorders, Fibrinolytic system

Micro-chirurgie et cataracte: technique d'extraction par petite incision 159 (V)

Microcirculation; prevention of experimental obstructions in the retinal microcirculation. Arterial fluorescein studies 345

Microscopie électronique, v. Homogenisates, Hornhautdystrophie, Irisnaht, Langerhans-Granula, Wound healing

Mikroaneurysmen, v. Exsudative Retinopathie, Microcirculation, Verfettung

Mikrochirurgie, v. Irisnaht, Juveniles Glaukom, Kongenitales Glaukom, Micro-chirurgie,

Pupillenerhaltung, Trabéculotomie, Trabekulektomie

Mikrophakie, v. Trisomie-13-Syndrom

Migraine; ophtalmodynamométrie et ophtalmodynamographie dans Thypo-tension orthostatique et les migraines $306(\mathrm{~V})$

Missbildungen, v. Juveniles Glaukom, Kongenitales Glaukom, Ophtalmo-plégie, Trisomie-13-

Syndrom

Mixed tumour; apocrine mixed tumour of the lid 548

Monokularsehen. v. Depth acuity

Müller'sche Stützzellen, v. Wound healing

Muscles oculaires, v. Ophtalmoplégie, Strabismus sursoadductorius

Mutation, v. Retinoblastoma

Myasthenie, v. Retinopathia pigmentosa

560

Index rerum ad Vol. 170

Myatrophie, neurogene, v. Retinopathia

pigmentosa Mycosis, v. Choroïdopathie maculaire,

Fusarium, Yeasts Mydriasis, v. Homer's syndrome Myopathie, v. Retinopathia pigmentosa

Narbenbildung, v. Wound healing 
Neovascularisation, v. Choroïdopathie maculaire

Nervus opticus, v. Endocarditis, Papillen-schwellungen, Sarkoidose, Vasculari-sation papillaire

Netzhaut, v. Adaptométrie, Choroïdo-pathie maculaire, Color angiography, Endocarditis,

Exsudative Retinopathie, Fibroplasie, Gasbrand, Microcircula-tion, Netzhautatrophie, Netzhaut-

funktionen, Pars planitis, Retinoschisis, Temperaturen, Thromben, Tuberculose miliaire, Wound healing

Netzhautatrophie; progressive Netzhautatrophie beim Hund 210 (V)

Netzhautf unktionen bei atypischer f amiliä-rer chorioretinaler Degeneration 223 (V)

Neurodermitis disseminata; kerato-konjunktivale Komplikationen bei Neurodermitis disseminata $183(\mathrm{~V})$

Neuro-Ophthalmologie, v. Gesichtsfeld-ausfälle, Horner's syndrome, Retinopathia pigmentosa, Sarkoidose

Non-contact tonometer, v. Tonomètre

Nystagmus, v. Equilibre

Obstruction capillaire, v. Micro-circulation

Ochse, v. Homogenisates

Ocular pressure, v. Propanololum, Tonomètre

Oedème maculaire, v. Pars planitis

Oedème papillaire, v. Papillen-schwellungen

Okulo-zerebro-renales Syndrom, v. Tri-somie-13-Syndrom

Operation, v. Adaptométrie, Iridectomie filtrante, Irisnaht, Juveniles Glaukom, Kongenitales

Glaukom, Micro-chirurgie, Pathologische Befunde,

Pupillenerhaltung, Strabismus surso-adductorius, Trabéculectomie, Trabéculotomie,

Trabekulektomie, Tumeurs multiples Ophtalmodynamographie, v. Migraine

Ophtalmodynamométrie, v. Migraine Ophtalmoplégie externe congénitale et familiale, aplasies musculaires et anomalies d'insertion 272 (V) Ophthalmoscope; a new indirect ophthalmoscope 56 Ophtotest, v. Examen automatique Opticus, v. Endocarditis, Papillen-schwellungen,

Sarkoidose, Vasculari-sation papillaire Opticusatrophie, v. Retinopathia pigmentosa,

Vascularisation papillaire Orbita, v. Tumeurs lymphoïdes, Tumeurs

multiples Orientierungshilfen, v. Hilfsmittel Outflow facility, v. Propanolum

Paedo-Ophthalmologie, v. Juveniles Glaukom, Kongenitales Glaukom, Ophtalmoplégie,

Retinoblastoma

Palsy, v. Equilibre, Ophtalmoplégie

Papille, v. Endocarditis, Papillen-schwellungen, Vascularisation papillaire

Papille de stase, v. Papillenschwellungen

Papillenschwellungen bei Leukaemien und verwandten Prozessen 494

Parese, v. Equilibre, Ophtalmoplégie

Parotis, v. Tumeurs lymphoïdes

Pars planitis; zur Entstehung des Makulaödems bei der Pars planitis 326

Pathologische Befunde; ausgewählte pathologische Befunde bei Trabekulektomie 108 (V)

Paupière, v. Langerhans-Granula, Mixed tumour, Tumeurs multiples, Yeasts

Perforation, v. Antibiotique

Perimetrie, v. Automatisierung, Gesichts-feldausfälle, Netzhautfunktionen

Périmétrie statique centrale, v. Adaptométrie

Index rerum ad Vol. 170

561 
Perméabilité capillaire, v. Dobésilate de

calcium, Exsudative Retinopathie Phenylephrin, v. Horner's syndrome Photocoagulation, v.

Adaptométrie,

Temperaturen, Thromben, Trabekulo-

punktur, Wound healing Pigmentary retinopathy, v. Retinopathia

pigmentosa Pigmentepithel, v. Wound healing Plasmazellen, v. Amyloid Platelet, v.

Microcirculation Polatest; Erfahrungen mit der Kombi-

nation von Polatest und Prismenvoll-

korrektion in der Behandlung von

Störungen des Binokularsehens

274 (V) Polymyalgia rheumatica, v. Temporal

arteritis Prepapillary abscess, v. Endocarditis Presbyopie, v. Mehrstärkenglas Prismen, v.

Polatest Proceedings, v. Schweizerische

Ophthalmologische Gesêilschaft Prolapsus de Гiris, v. Pupillenerhaltung Propanololum; emploi

clinique et

mécanisme d'action du propanololum

36 Protein, v. Temporal arteritis, Trans-

ferrine 32P-Test; die Anwendung von Halbleiter-

detektoren beim 32P-Test 51 Ptosis, v. Ophtalmoplégie Pupille, v. Horner's syndrome

Pupillenerhaltung; Eingriffe an der Iris

zur Erhaltung oder Wiederherstellung

einer runden Pupille 156 (V)

Rabbit, v. Anesthesia, Homogenisates, Irisnaht, Transferrine, Wound healing

Reading aid, v. Hilfsmittel

Recherches expérimentales, v. Anesthesia, Depth acuity, Homogenisates, Irisnaht,

Microcirculation, Tolerance, Trabe-kulopunktur, Transferrine, Wound healing, Yeasts

Refraktion, v. Mehrstärkenglas, Polatest

Regeneration, v. Homogenisates

Resistance capillaire, v. Circulatory disorders, Dobésilate de calcium, Medikamentöse Therapie

Retina, v. Adaptométrie, Choroïdopathie maculaire, Color angiography, Endocarditis,

Exsudative Retinopathie, Fibroplasie, Gasbrand, Micro-circulation, Netzhautatrophie, Netz-

hautfunktionen, Pars planitis, Retino-schisis, Temperaturen, Thromben, Tuberculose miliaire,

Wound healing

Retinal detachment, v. Adaptométrie, Retinoschisis

Retinitis, v. Endocarditis

Retinoblastoma; genesis and genetics of retinoblastoma 405

Retinopathia diabetica, v. Circulatory disorders, Dobésilate de calcium, Exsudative Retinopathie, Fibrinolytic system, Medikamentöse Therapie, Microcirculation, Verfettung

Retinopathia pigmentosa; klinisch-neurologische und elektromyo-graphische Untersuchungen

bei Kranken mit anscheinend isolierter Retinopathia pigmentosa 505

Retinoschisis; ein neues morphisches Zeichen zur Differentialdiagnose der senilen Retinoschisis $244(\mathrm{~V})$

Rods, v. Netzhautfunktionen

Roth's spots, v. Endocarditis

Sarkoidose; generalisierte Sarkoidose mit 
Gehirn- und Augenbeteiligung 393 Savons, v. Anesthesia Scar formation, v. Wound healing Schielen, v. Polatest, Strabismus

sursoadductorius Schweissdrüse, v. Mixed tumour Schweizerische Ophthalmologische

Gesêilschaft. 66. Generalversammlung,

Montreux 1973, 85-312 (V) Schwerhörigkeit, v. Retinopathia

pigmentosa Sehschärfe, v. Gesichtsfeldausfälle Seifen, v. Anesthesia

562

Index rerum ad Vol. 170

Sensibilité cornéenne, v. Anesthesia Skotom, v. Zentralskotom Soaps, v. Anesthesia Société Suisse d'Ophtalmologie. 66e Assemblée générale, Montreux 1973, 85-312 (V) Soft lens; die «therapeutische soft lens»,

300 (V) Spinaliom, v. Langerhans-Granula Squint, v. Polatest, Strabismus surso-

adductorius Stäbchen, v. Netzhautfunktionen Static central field, v. Adaptométrie

Stauungspapille, v. Papillenschwellungen Stereopsis. v. Depth acuity, Equilibre,

Polatest Steroide, v. Yeasts

Stoffwechsel, v. Retinopathia pigmentosa Strabisme, v. Polatest, Strabismus sursoadductorius Strabismus sursoadductorius; pseudo-einseitiger Strabismus sursoadductorius 283

(V) Suppression, v. Polatest Surdité, v. Retinopathia pigmentosa Surgery, v. Adaptométrie, Iridectomie filtrante, Irisnaht, Juveniles Glaukom, Kongenitales Glaukom, Micro-chirurgie, Pathologische Befunde, Pupillenerhaltung, Strabismus sursoadductorius, Trabeculectomie, Trabé-culotomie, Trabekulektomie, Tumeurs multiples Sweat gland, v. Mixed tumour

Sympathicus, v. Horner's syndrome Syndrome, v. Horner's syndrome, Retinopathia pigmentosa, Trisomie-13-Syndrom

Tapetum lucidum, v. Netzhautatrophie Temperaturen; Abschätzung der Tem-

peraturen in bestrahlten retinalen

Gefässen 251 (V) Temporal arteritis; amyloid deposits of

internal elastic lamina in temporal

arteritis 337 Tension artérielle, v. Migraine

Tension oculaire, v. Propanololum,

Tonometre Test, v. Diagnostic étiologique, Examen

automatique, Polatest, 32P-Test Therapie, v. Antibiotique, Circulatory

disorders, Dobésilate de calcium,

Endocarditis, Fusarium, Juveniles

Glaukom, Langzeittherapie, Medika-

mentöse Therapie, Papillenschwellungen, Polatest, Propanololum, Soft

lens, Yeasts Thromben; experimentelle Erzeugung

von Thromben durch Bestrahlung mit

intensivem Licht 252 (V) Thrombolysis, v. Fibrinolytic system Thrombozyt, v. Microcirculation

Thymidine au tritium, v. Wound healing Tiefenschärfe, v. Depth acuity Tolerance aux

antimitotiques introduits

dans la chambre antérieure de $\Gamma$ ceil

du chat 199 (V) Tonometre; le tonometre à air 446 Trabeculectomie; la trabeculectomie, nos premiers résultats $130(\mathrm{~V})$ Trabéculotomie; indications et

résultats de la trabéculotomie 
ab externo 124 (V) Trabekulektomie; Ergebnisse der Trabekulektomie. Auswertung von 99 Fallen

133 (V) Trabekulopunktur; zur Laser-Trabekulo-

punktur. I. Experimentelle Unter-

suchungen 362 Trabekulotomie, v. Kongenitales

Glaukom, Trabéculotomie Tränendrüse, v. Tumeurs lymphoïdes Tränenwege, v. Yeasts

Transferrine; la transferrine des liquides

endoculaires chez le lapin 72 Trauma, v. Antibiotique, Fusarium Trepanation selon Elliott, v.

Juveniles

Glaukom Trisomie-13-Syndrom; Buphthalmus bei

Trisomie-13-Syndrom 381 Tuberculose miliaire choroïdienne dissé-

minée. Etude angiofluorographique

$206(\mathrm{~V})$

Index rerum ad Vol. 170

563

Tuberkulin-Anergie, v. Choroïdopathie maculaire

Tumeurs intra-oculaires; limites et causes d'erreur de Гexamen échographique (méthode A) dans le diagnostic des tumeurs intra-oculaires 201 (V)

Tumeurs lymphoïdes; les tumeurs lymphoïdes de Torbite 290 (V)

Tumeurs multiples de la sphere oculaire 293 (V)

Tumor, v. Langerhans-Granula, Meta-static tumour, Mixed tumour, Papillen-schwellungen, 32P-

Test, Retino-blastoma, Tumeurs intra-oculaires, Tumeurs lymphoïdes, Tumeurs multiples

Tumors; orbital tumors 392 (B)

Usher-Syndrom, v. Retinopathia

pigmentosa Uveitis, v. Diagnostic étiologique,

Immunologie, Sarkoidose

Vaisseaux, v. Choroïdopathie maculaire, Circulatory disorders, Color angio-graphy, Dobésilate de calcium, Exsu-dative Retinopathie, Fibrínolytic system, Gasbrand, Medikamentöse Therapie, Microcirculation, Pars planitis, Temperaturen, Temporal arteritis, Thromben, Vascularisation papillaire, Verfettung

Varia 476

Vascularisation papillaire; etude de la vascularisation papillaire au moyen de Гangiographie fluorescéinique $254(\mathrm{~V})$

Verbrennung, v. Homogenisates

Vererbung, v. Amyloid, Netzhautatrophie, Netzhautfunktionen, Neurodermitis disseminata,

Ophtalmoplégie, Retino-blastoma, Retinopathia pigmentosa, Temporal arteritis

Verfettung der Mikroaneurysmen bei Retinopathia diabetica 229 (V)

Verhandlungsberichte, v. Schweizerische Ophthalmologische Gesellschaft

Verre progressif, v. Mehrstärkenglas

Verruca, v. Langerhans-Granula

Vessels, v. Choroïdopathie maculaire, Circulatory disorders, Color angio-graphy, Dobésilate de calcium, Ex-sudative Retinopathie, Fibrinolytic system, Gasbrand, Medikamentöse Therapie, Microcirculation, Pars planitis, Temperaturen, Temporal arteritis, Thromben, Vascularisation papillaire, Verfettung

Visual acuity, v. Gesichtsfeldausfälle 
Visual field, v. Automatisierung, Gesichtsfeldausfälle, Netzhautfunktionen

Vision binoculaire, v. Depth acuity, Equilibre, Polatest

Vision des couleurs, v. Dyschromatopsy

Visus, v. Gesichtsfeldausfälle

Vitrektomie $172(\mathrm{~V})$

Vitreous body, v. Fibroplasie, Pars planitis, Vitrektomie

Voies lacrymales, v. Yeasts

Vorderkammer, v. Tolerance, Vorderkammerlinsen

Vorderkammerlinsen; erste Erfahrungen mit der Einpflanzung von Vorderkammerlinsen (Iris-

Clip-Linsen nach Binkhorst) 165 (V)

Wound healing after xenon arc photo-coagulation in the rabbit retina. Identification of the proliferating cells in the lesion by light and electron microscopic autoradiography using $3 \mathrm{H}-$

Thymidine 462

Yeasts in banal external ocular inflammations 13

Zapfen, v. Netzhautfunktionen

Zentralskotom; akkommodatives Ver-halten von Augen mit organisch be-dingtem Zentralskotom $269(\mathrm{~V})$

Z. Ziliarkörper

v. Metastatic tumour

Sarkoidose

Zystoide Degeneration

v. Retinoschisis 